Article

\title{
The Fractional Orthogonal Derivative
}

\section{Enno Diekema}

Kooikersdreef 620, 7328 BS Apeldoorn, The Netherlands; E-Mail: e.diekema@gmail.com; Tel.: 06-14115525

Academic Editor: Hari M. Srivastava

Received: 4 March 2015 / Accepted: 10 April 2015 / Published: 22 April 2015

\begin{abstract}
This paper builds on the notion of the so-called orthogonal derivative, where an $n$-th order derivative is approximated by an integral involving an orthogonal polynomial of degree $n$. This notion was reviewed in great detail in a paper by the author and Koornwinder in 2012. Here, an approximation of the Weyl or Riemann-Liouville fractional derivative is considered by replacing the $n$-th derivative by its approximation in the formula for the fractional derivative. In the case of, for instance, Jacobi polynomials, an explicit formula for the kernel of this approximate fractional derivative can be given. Next, we consider the fractional derivative as a filter and compute the frequency response in the continuous case for the Jacobi polynomials and in the discrete case for the Hahn polynomials. The frequency response in this case is a confluent hypergeometric function. A different approach is discussed, which starts with this explicit frequency response and then obtains the approximate fractional derivative by taking the inverse Fourier transform.
\end{abstract}

Keywords: orthogonal derivative; orthogonal polynomials; hypergeometric functions; Fourier transform; frequency response

\section{Introduction}

As soon as differential calculus was invented by Newton and Leibniz, the question arose about the meaning of a derivative of non-integer order. Many mathematicians have worked on the subject, and many different definitions were proposed.

An excellent historical survey is given in the book [1]. In the encyclopedic work [2], most of the usual definitions are given. The book [3] give a survey of a number of definitions of the fractional calculus. 
The book [4] distinguishes fractional derivatives of causal functions, as well as non-causal functions. Last, but not least, [5] give a survey of the developments in the field from 1974.

Nowadays, fractional derivatives are much used in fractional differential equations, which are very suitable for describing certain physical phenomena, better than when using ordinary derivatives. For instance, a fractional PID (proportional, integrating and differentiating) controller can be made much simpler and more robust than an ordinary PID controller. Some other areas of application of the fractional derivatives are viscoelastic materials, hydrodynamics, rheology, diffusive transport, electrical networks, control theory, electromagnetic theory, signal and image processing and probability. The definition of fractional derivative may vary with the application, since in each case, one will look for the most suitable definition.

Unlike the ordinary derivative, there is no simple geometric description of the fractional derivative. However, see [6,7].

The most common definitions of fractional derivative, which we also use in this paper, are by taking ordinary derivatives of a Riemann-Liouville or Weyl fractional integral. Even here, different choices can be made, which give quite different results. For instance, with $\nu>0$ and $\mu=n-\nu>0$, we can write:

$$
\frac{d^{\nu}}{d x^{\nu}} e^{x}=\frac{d^{n}}{d x^{n}} \frac{1}{\Gamma(\mu)} \int_{-\infty}^{x}(x-y)^{\mu-1} e^{y} d y=e^{x}=\sum_{k=0}^{\infty} \frac{x^{k}}{\Gamma(k+1)}
$$

and, thus, obtain a natural choice for the fractional derivative of $e^{x}$, but we can also choose the definition:

$$
\frac{d^{\nu}}{d x^{\nu}} e^{x}=\frac{d^{n}}{x^{n}} \frac{1}{\Gamma(\mu)} \int_{0}^{x}(x-y)^{\mu-1} e^{y} d y=\sum_{k=0}^{\infty} \frac{x^{k-\nu}}{\Gamma(k-\nu+1)}
$$

Here, the last identity follows from:

$$
\frac{d^{\nu}}{d x^{\nu}} x^{k}=\frac{d^{n}}{d x^{n}} \frac{1}{\Gamma(\mu)} \int_{0}^{x}(x-y)^{\mu-1} y^{k} d y=\frac{\Gamma(k+1)}{\Gamma(k-\nu+1)} x^{k-\nu}
$$

which looks like a natural choice for the fractional derivative of $x^{k}$. (note that replacement of the lower bound of the last integral by $-\infty$ would give a divergent integral). Thus, one has to be careful with just extrapolating a natural looking definition of a fractional derivative of some elementary functions. Our definitions follow Miller and Ross [1].

In [8], we reviewed a formula for the so-called orthogonal derivative, which has a long history. This derivative (of order $n$ ) can be computed as a limit of a certain integral. This is a generalization of the usual notion of the $n$-th order derivative, and more importantly, when ignoring the limit, it can be used as an approximation of the $n$-th order derivative. In the present paper, the orthogonal derivative will be used in order to generalize the definitions of Riemann-Liouville and Weyl for the fractional derivative and to approximate these fractional derivatives. In the case of the orthogonal derivative associated with the Jacobi polynomials, the kernel of the resulting integral transform approximating the fractional derivative can be computed explicitly. In the case of the Hahn polynomials, similar explicit results are obtained for the approximation of the fractional difference.

In general, Fourier and Laplace transforms are important tools for finding solutions of fractional orthogonal differential equations. Therefore, in our paper, we also consider the action of the various operators in the frequency domain. We consider the fractional derivative as a filter with a so-called 
frequency response. Instead of a convolution, we then have multiplication of transfer functions. A picture of the modulus of the frequency response gives very good insight into how well the approximating operators behave.

The idea of combining an orthogonal derivative with a fractional integral in order to approximate fractional derivatives is already discussed in $[9,10]$. However, explicit formulas, like ours are not given in these papers. Furthermore, a discussion of the filter in the frequency domain is missing.

Let us now give a summary of the sections of this paper.

In Section 2, the definitions and basic properties of the Riemann-Liouville and Weyl fractional integral and derivative are recalled. Furthermore, their Fourier transforms are mentioned.

In Section 3, the approximate Weyl and Riemann-Liouville fractional derivatives are defined by using the approximate $n$-th order derivative coming from the orthogonal derivative.

In Section 4, we give explicit expressions in the case of the Jacobi polynomials. These simplify in the case of Gegenbauer and Legendre polynomials.

In Section 5, we consider the frequency response of the approximate fractional derivative. Explicit results in the continuous case are given for Jacobi polynomials and in the discrete case for Hahn polynomials.

The general case suggests an alternative approach to obtain an approximate fractional derivative, by starting with a frequency response, which approximates the frequency response of the fractional derivative, and then taking the inverse Fourier transform.

This method is discussed in Section 6 for a frequency response given by a confluent hypergeometric function, and in Section 7, there are two more examples involving elementary transfer functions.

We also studied the fractional orthogonal difference with application to a discrete filter. This will appear in the next paper [11].

Remark 1.1. For $\nu \in \mathbb{C}$, the power $z^{\nu}$ will always be considered as a function of $z$ in the complex plane with cut $(-\infty, 0]$, i.e., $z^{\nu}=e^{\nu \ln z}$ with $z \neq 0$ and $-\pi<\arg (z)<\pi$. On the cut, for $x \in(-i y, 0)$, we distinguish between $(x+i 0)^{\nu}=\lim _{y \downarrow 0}(x+i y)^{\nu}=e^{i \pi \nu}(-x)^{\nu}$ and $(x-i 0)^{\nu}=\lim _{y \downarrow 0}(x-i y)^{\nu}=$ $e^{-i \pi \nu}(-x)^{\nu}$. For $y \in(R) \backslash\{0\}$, the convention applies to $(i y)^{\nu}$, but sometimes, we write for clarity $(i y)^{\nu}=(i y+0)^{\nu}=\lim _{x \downarrow 0}(i y+x)^{\nu}$.

\section{The Riemann-Liouville and the Weyl Fractional Transforms}

We define two versions of the fractional integral: the Riemann-Liouville and the Weyl transform. In terms of these, we can define a fractional derivative.

Let $\mu \in \mathbb{C}$ with $\operatorname{Re}(\mu)>0$. Let $f$ be a function on $(-\infty, b)$, which is integrable on bounded subintervals. Then, the Riemann-Liouville integral of order $\mu$ is defined as:

$$
R^{-\mu}[f](x)=f^{(-\mu)}(x):=\frac{1}{\Gamma(\mu)} \int_{-\infty}^{x} f(y)(x-y)^{\mu-1} d y
$$


A sufficient condition for the absolute convergence of this integral is $f(-x)=O\left(x^{-\mu-\varepsilon}\right)$, $\varepsilon>0, x \rightarrow \infty$. Similarly, for $\operatorname{Re}(\mu)>0$ and $f$, a locally-integrable function on $(a, \infty)$, such that $f(x)=O\left(x^{-\mu-\varepsilon}\right), \varepsilon>0, x \rightarrow \infty$, the Weyl integral of order $\mu$ is defined as:

$$
W^{-\mu}[f](x)=f^{(-\mu)}(x):=\frac{1}{\Gamma(\mu)} \int_{x}^{\infty} f(y)(y-x)^{\mu-1} d y
$$

Clearly:

$$
R^{-\mu}[f(-.)](-x)=W^{-\mu}[f](x)
$$

The Riemann-Liouville integral is often given for a function $f$ on $[0, b)$ in the form:

$$
R^{-\mu}[f](x)=f^{(-\mu)}(x):=\frac{1}{\Gamma(\mu)} \int_{0}^{x} f(y)(x-y)^{\mu-1} d y \quad(x>0)
$$

This can be obtained from (1) if we assume that $f(x)=0$ for $x \leq 0$ (then, $f$ is called a causal function). For instance, if $f(x)=x^{\alpha} \quad(x>0)$ and $f(x)=0 \quad(x \leq 0)$, then:

$$
R^{-\mu}[f](x)=\frac{\Gamma(\alpha+1)}{\Gamma(\alpha+\mu+1)} x^{\alpha+\mu} \quad(x>0, \operatorname{Re}(\alpha)>-1)
$$

and

$$
W^{-\mu}[f](x)=\frac{\Gamma(-\alpha-\mu)}{\Gamma(-\alpha)} x^{\alpha+\mu} \quad(x>0, \operatorname{Re}(\alpha+\mu)<0)
$$

When $-1<\operatorname{Re}(\alpha)<\operatorname{Re}(-\mu)<0$, both formulas are valid. The quotients of Gamma functions are related by:

$$
\frac{\Gamma(-\alpha-\mu)}{\Gamma(-\alpha)}=\frac{\sin (\alpha \pi)}{\sin (\alpha+\mu) \pi} \frac{\Gamma(\alpha+1)}{\Gamma(\alpha+\mu+1)}
$$

Substitution in (2) gives:

$$
W^{-\mu}[f](x)=\frac{\sin (\alpha \pi)}{\sin (\alpha+\mu) \pi} R^{-\mu}[f](x)
$$

For $\operatorname{Re}(\nu)<n, n$ a positive integer and $D=\frac{d}{d x}$, one can easily derive:

$$
\begin{gathered}
R^{\nu}[f](x)=D^{n}\left[R^{\nu-n}[f]\right](x) \\
W^{\nu}[f](x)=(-1)^{n} D^{n}\left[W^{\nu-n}[f]\right](x)
\end{gathered}
$$

For $0<\operatorname{Re}(\nu)<n$, we define the fractional derivative by (3) and (4). These are certainly well defined if all derivatives of $f$ up to order $n$ exist. The definition is independent of the choice $n>\operatorname{Re}(\nu)$.

Put $W^{0}=i d=R^{0}$. Under the assumption of sufficient differentiability and convergence, we have $W^{\mu} W^{\nu}=W^{\mu+\nu}, R^{\mu} R^{\nu}=R^{\mu+\nu}$ for all $\mu, \nu \in \mathbb{C}$ and $R^{n}=D^{n}, W^{n}=(-1)^{n} D^{n}$.

Because we need the Fourier transform of (3) and (4), we use the following theorem proven in [2] (Chapter 2 §7) for the Riemann-Liouville derivative, as well for the Weyl derivative.

Theorem 2.1. Let $f$ and $g$ be functions on $\mathbb{R}$ for which the Fourier transforms exist and are given by:

$$
F(\omega)=\frac{1}{\sqrt{2 \pi}} \int_{-\infty}^{\infty} e^{i \omega x} f(x) d x
$$




$$
G(\omega)=\frac{1}{\sqrt{2 \pi}} \int_{-\infty}^{\infty} e^{i \omega x} g(x) d x
$$

If $g=R^{\nu}[f]$, then the following relation holds:

$$
H(\omega)=\frac{G(\omega)}{F(\omega)}=(-i \omega)^{\nu}
$$

If $g=W^{\nu}[f]$, then:

$$
H(\omega)=\frac{G(\omega)}{F(\omega)}=(i \omega)^{\nu}
$$

$H(\omega)$ will be called the frequency response, where we follow the terminology of filter theory.

\section{The Fractional Weyl Transform for the Orthogonal Derivative}

In [8] (Theorem 3.1), the following theorem is proved (see [8], Section 2.1, for generalities about orthogonal polynomials):

Theorem 3.1. Let $n$ be a positive integer. Let $p_{n}$ be an orthogonal polynomial of degree $n$ with respect to the orthogonality measure $\mu$ for which all moments exist. Let $x \in \mathbb{R}$. Let I be a closed interval containing $x$, such that $x+\delta \operatorname{supp}(\mu) \subset I$ for $\delta>0$ small enough. Let $f$ be a continuous function on $I$, such that its derivatives of order $1,2, \ldots, n$ at $x$ exist (right or left derivatives if $x$ is the boundary point of I). In addition, if I is unbounded, assume that $f$ is of at most polynomial growth on I. Then:

$$
f^{(n)}(x)=\lim _{\delta \downarrow 0} D_{\delta}^{n}[f](x)
$$

where:

$$
D_{\delta}^{n}[f](x)=\frac{k_{n} n !}{h_{n}} \frac{1}{\delta^{n}} \int_{\mathbb{R}} f(x+\delta \xi) p_{n}(\xi) d \mu(\xi)
$$

By the assumptions, the integral converges absolutely. $h_{n}$ and $k_{n}$ are defined by:

$$
h_{n}=\int_{\mathbb{R}} p_{n}(\xi)^{2} d \mu(\xi)
$$

and

$$
p_{n}(x)=k_{n} x^{n}+q_{n-1}(x)
$$

with $q_{n-1}$ a polynomial of degree $<n$. We call the limit given by the right-hand side of (6) the orthogonal derivative at $x$ of order $n$. See Remark 3.3 for some weaker conditions.

Theorem 3.1 suggests the definition of the approximate fractional orthogonal derivative. We can apply (6) in (4). This yields:

$$
W^{\nu}[f](x)=\frac{(-1)^{n}}{\Gamma(n-\nu)} \frac{d^{n}}{d x^{n}}\left[\int_{x}^{\infty} f(y)(y-x)^{n-\nu-1} d y\right]=\lim _{\delta \downarrow 0} W_{\delta}^{\nu, n}[f](x)
$$

where:

$$
W_{\delta}^{\nu, n}[f](x):=(-1)^{n} D_{\delta}^{n}\left[W^{\nu-n}[f]\right](x)=(-1)^{n} W^{\nu-n}\left[D_{\delta}^{n}[f]\right](x)
$$

We call $W_{\delta}^{\nu, n}[f]$ the approximate fractional orthogonal derivative. The existence of sufficiently many derivatives of $f$ is no longer required for the definition (9) to be valid if $\nu>0$. However, for the 
definition of $W^{\nu}[f]$ and for the validity of the limit in (8), sufficient differentiability is still required. The last equality in (9) follows from the fact that the operators $W^{\nu-n}$ and $D_{\delta}^{n}$ are convolution operators (i.e., operators that commute with translations), and therefore, the integrals can be interchanged. Then, it follows by substitution of (7):

$$
\begin{aligned}
W_{\delta}^{\nu, n}[f](x) & =\frac{(-1)^{n} k_{n} \Gamma(n+1)}{h_{n} \Gamma(n-\nu)} \frac{1}{\delta^{n}} \int_{-\infty}^{\infty}\left[\int_{x+\delta u}^{\infty} f(y)(y-x-\delta u)^{n-\nu-1} d y\right] p_{n}(u) d \mu(u)= \\
& =\frac{(-1)^{n} k_{n} \Gamma(n+1)}{h_{n} \Gamma(n-\nu)} \frac{1}{\delta^{n}} \int_{-\infty}^{\infty}\left[\int_{x}^{\infty} f(y+\delta u)(y-x)^{n-\nu-1} d y\right] p_{n}(u) d \mu(u)
\end{aligned}
$$

With $y=x+\delta(s-u)$, it follows:

$$
W_{\delta}^{\nu, n}[f](x)=\frac{(-1)^{n} k_{n} \Gamma(n+1)}{h_{n} \Gamma(n-\nu)} \frac{1}{\delta^{\nu}} \int_{-\infty}^{\infty}\left[\int_{u}^{\infty} f(x+\delta s)(s-u)^{n-\nu-1} d s\right] p_{n}(u) d \mu(u)
$$

Now, we consider three typical cases for the orthogonality interval:

- Finite orthogonality interval $[-1,1]$.

- Infinite orthogonality interval $[0, \infty)$.

- Infinite orthogonality interval $(-\infty, \infty)$.

Finite orthogonality interval $[-1,1]$ :

The integral (10) can be split up into two integrals as:

$$
\begin{aligned}
W_{\delta}^{\nu, n}[f](x) & =\frac{(-1)^{n} k_{n} \Gamma(n+1)}{h_{n} \Gamma(n-\nu)} \frac{1}{\delta^{\nu}} \int_{-1}^{1}\left[\int_{u}^{1} f(x+\delta y)(y-u)^{n-\nu-1} d y\right] p_{n}(u) d \mu(u)+ \\
& +\frac{(-1)^{n} k_{n} \Gamma(n+1)}{h_{n} \Gamma(n-\nu)} \frac{1}{\delta^{\nu}} \int_{-1}^{1}\left[\int_{1}^{\infty} f(x+\delta y)(y-u)^{n-\nu-1} d y\right] p_{n}(u) d \mu(u)
\end{aligned}
$$

Interchanging the double integrals in both terms gives:

$$
\begin{aligned}
W_{\delta}^{\nu, n}[f](x) & =\frac{(-1)^{n} k_{n} \Gamma(n+1)}{h_{n} \Gamma(n-\nu)} \frac{1}{\delta^{\nu}} \int_{-1}^{1} f(x+\delta y)\left[\int_{-1}^{y} p_{n}(u)(y-u)^{n-\nu-1} d \mu(u)\right] d y+ \\
& +\frac{(-1)^{n} k_{n} \Gamma(n+1)}{h_{n} \Gamma(n-\nu)} \frac{1}{\delta^{\nu}} \int_{1}^{\infty} f(x+\delta y)\left[\int_{-1}^{1} p_{n}(u)(y-u)^{n-\nu-1} d \mu(u)\right] d y
\end{aligned}
$$

Infinite orthogonality interval $[0, \infty)$ :

The outer integral in (10) is now from zero to $\infty$. Interchanging the double integral gives:

$$
W_{\delta}^{\nu, n}[f](x)=\frac{(-1)^{n} k_{n} \Gamma(n+1)}{h_{n} \Gamma(n-\nu)} \frac{1}{\delta^{\nu}} \int_{0}^{\infty} f(x+\delta y)\left[\int_{0}^{y}(y-u)^{n-\nu-1} p_{n}(u) d \mu(u)\right] d y
$$

Infinite orthogonality interval $(-\infty, \infty)$ :

The outer integral in (10) is now from $-\infty$ to $\infty$. Interchanging the double integral gives:

$$
W_{\delta}^{\nu, n}[f](x)=\frac{(-1)^{n} k_{n} \Gamma(n+1)}{h_{n} \Gamma(n-\nu)} \frac{1}{\delta^{\nu}} \int_{-\infty}^{\infty} f(x+\delta y)\left[\int_{-\infty}^{y}(y-u)^{n-\nu-1} p_{n}(u) d \mu(u)\right] d y
$$

In (11), (12) and (13), the integrals inside the square brackets can be expected to be computable analytically or numerically for concrete measures $\mu$ and orthogonality polynomials $p_{n}$. 
Remark 3.2. We have derived these formulas for all values of $\nu$ with $\operatorname{Re}(\nu)<n$, but our main usage of them will be for $\operatorname{Re}(\nu) \geq 0$, where they are approximate fractional derivatives.

Remark 3.3. In this paper, we assume for simplicity that the orthogonal derivative is equal to the ordinary derivative, i.e., that both sides of (6) are well defined and equal, which is certainly true under the assumptions of Theorem 3.1. It should be noted that the definition of the orthogonal derivative is valid for a wider class of functions than the ordinary derivative. Therefore, if the limit of the right-hand side of (6) exists and $f^{(n)}(x)$ does not exist, we still call the right-hand side of (6) the orthogonal derivative. For example, the ordinary derivative does not exist for the function $f(x)=|x|$ for $x=0$, but the orthogonal derivative for this function does exist. For less trivial examples, see [8] (Section 3.8).

Remark 3.4. Instead of the Weyl integral, we could have worked with the Riemann-Liouville integral. For example, the following formulas can be obtained:

$$
R_{\delta}^{\nu, n}[f](x)=R^{\nu-n}\left[D_{\delta}^{n}[f]\right](x) \quad R^{\nu}[f](x)=\lim _{\delta \downarrow 0} R_{\delta}^{\nu, n}[f](x)
$$

For the orthogonality interval $[-1,1]$, it follows:

$$
\begin{aligned}
R_{\delta}^{\nu, n}[f](x) & =\frac{k_{n} \Gamma(n+1)}{h_{n} \Gamma(n-\nu)} \frac{1}{\delta^{\nu}} \int_{-1}^{1} f(x+\delta y)\left[\int_{y}^{1}(u-y)^{n-\nu-1} p_{n}(u) d \mu(u)\right] d y+ \\
& +\frac{k_{n} \Gamma(n+1)}{h_{n} \Gamma(n-\nu)} \frac{1}{\delta^{\nu}} \int_{-\infty}^{-1} f(x+\delta y)\left[\int_{-1}^{1}(u-y)^{n-\nu-1} p_{n}(u) d \mu(u)\right] d y
\end{aligned}
$$

For the orthogonality interval $(-\infty, \infty)$, it follows:

$$
R_{\delta}^{\nu, n}[f](x)=\frac{k_{n} \Gamma(n+1)}{h_{n} \Gamma(n-\nu)} \frac{1}{\delta^{\nu}} \int_{-\infty}^{\infty} f(x+\delta y)\left[\int_{y}^{\infty}(u-y)^{n-\nu-1} p_{n}(u) d \mu(u)\right] d y
$$

All results in this paper could also have been equivalently formulated in terms of $R_{\delta}^{\nu, n}$.

\section{The Fractional Weyl Transform for the Jacobi Derivative}

In this section, we apply (11) to the Jacobi polynomials, where:

$$
\begin{gathered}
p_{n}(x)=P_{n}^{(\alpha, \beta)}(x) \\
d \mu(x)=w(x) d x=(1-x)^{\alpha}(1+x)^{\beta} d x \quad \alpha>-1 \quad \beta>-1 \\
\frac{h_{n}}{k_{n}}=2^{n+\alpha+\beta+1} \frac{\Gamma(n+\alpha+1) \Gamma(n+\beta+1)}{\Gamma(2 n+\alpha+\beta+2)} \\
P_{n}^{(\alpha, \beta)}(-x)=(-1)^{n} P_{n}^{(\beta, \alpha)}(x)
\end{gathered}
$$

For the resulting transform, which we call the approximate fractional Jacobi derivative, we write $W_{\delta}^{\nu, n}=W_{\delta, \alpha, \beta}^{\nu, n}$. Before computing this transform, we observe the following proposition:

Proposition 4.1. For the approximate fractional Jacobi derivative, the following formula is valid:

$$
W_{\delta, \alpha, \beta}^{\nu, n}[f](x)=W_{\delta, \alpha+1, \beta+1}^{\nu, n-1}[f](x)
$$

Hence, by iteration, $W_{\delta, \alpha, \beta}^{\nu, n}[f](x)=W_{\delta, \alpha+n-m, \beta+n-m}^{\nu, m}[f](x)$, where $m$ is the smallest integer $>\nu$. 
Proof. From [8] (3.10), it follows that:

$$
D_{\delta, \alpha, \beta}^{n}[f](x)=\frac{\int_{-1}^{1} f^{(n)}(x+\delta \xi)(1-\xi)^{n+\alpha}(1+\xi)^{n+\beta} d \xi}{\int_{-1}^{1}(1-\xi)^{n+\alpha}(1+\xi)^{n+\beta} d \xi}=D_{\delta, \alpha+1, \beta+1}^{n-1}\left[f^{\prime}\right](x)
$$

From (4), we obtain in a straightforward way:

$$
\begin{aligned}
W_{\delta, \alpha, \beta}^{\nu, n}[f](x) & =(-1)^{n} D_{\delta, \alpha, \beta}^{n}\left[W^{\nu-n}[f]\right](x)= \\
& =(-1)^{n} D_{\delta, \alpha, \beta}^{n-1}\left(W^{\nu-n}[f]\right)^{\prime}(x)= \\
& =(-1)^{n-1} D_{\delta, \alpha+1, \beta+1}^{n-1}\left[W^{\nu-n+1}[f]\right](x)=W_{\delta, \alpha+1, \beta+1}^{\nu, n-1}[f](x)
\end{aligned}
$$

Remark 4.2. For the Laguerre and Hermite derivatives, there are similar formulas. The formula for the Hermite derivative has no parameters.

For the computation of $W_{\delta, \alpha, \beta}^{\nu, n}$, we substitute (14)-(17) in (11).

$$
W_{\delta, \alpha, \beta}^{\nu, n}[f](x)=\frac{(-1)^{n} \Gamma(2 n+\alpha+\beta+2)}{2^{n+\alpha+\beta+1} \Gamma(n+\alpha+1) \Gamma(n+\beta+1)} \frac{\Gamma(n+1)}{\Gamma(n-\nu)} \frac{1}{\delta^{\nu}}\left[I_{1}(x)+I_{2}(x)\right]
$$

where:

$$
\begin{aligned}
& I_{1}(x)=\int_{-1}^{1} f(x+\delta y) J_{1}(y) d y \\
& I_{2}(x)=\int_{1}^{\infty} f(x+\delta y) J_{2}(y) d y
\end{aligned}
$$

with:

$$
\begin{array}{ll}
J_{1}(y)=\int_{-1}^{y} P_{n}^{(\alpha, \beta)}(u)(1-u)^{\alpha}(1+u)^{\beta}(y-u)^{n-\nu-1} d u & -1 \leq y \leq 1 \\
J_{2}(y)=\int_{-1}^{1} P_{n}^{(\alpha, \beta)}(u)(1-u)^{\alpha}(1+u)^{\beta}(y-u)^{n-\nu-1} d u & 1 \leq y \leq \infty
\end{array}
$$

Note that $W_{\delta, \alpha, \beta}^{\nu, n}[f](x)$ is analytic in $\nu$ for $\operatorname{Re}(\nu)<n$. First, we assume that $\operatorname{Re}(\nu)<0$.

For the computation of $J_{1}(y)$, the Rodrigues formula for Jacobi polynomials is used:

$$
P_{n}^{(\alpha, \beta)}(u)=\frac{1}{(-1)^{n} 2^{n} n !(1-u)^{\alpha}(1+u)^{\beta}} \frac{d^{n}}{d u^{n}}\left[(1-u)^{n+\alpha}(1+u)^{n+\beta}\right]
$$

Substitution in (21) yields:

$$
J_{1}(y)=\frac{(-1)^{n}}{2^{n} n !} \int_{-1}^{y} \frac{d^{n}}{d u^{n}}\left[(1-u)^{n+\alpha}(1+u)^{n+\beta}\right](y-u)^{n-\nu-1} d u
$$

Repeated integration by parts ( $n$ times) gives:

$$
J_{1}(y)=\frac{(-1)^{n}}{2^{n} n !} \frac{\Gamma(n-\nu)}{\Gamma(-\nu)} \int_{-1}^{y}(1-u)^{n+\alpha}(1+u)^{n+\beta}(y-u)^{-\nu-1} d u
$$


Substitution of the variable $u=(1+y) w-1$, using [12] (Theorem 2.2.1) and [13] (15.8.1) in the third identity gives:

$$
\begin{aligned}
J_{1}(y) & =(-1)^{n} 2^{\alpha}(1+y)^{n+\beta-\nu} \frac{\Gamma(n+\beta+1) \Gamma(n-\nu)}{n ! \Gamma(n-\nu+\beta+1)} F\left(\begin{array}{c}
n+\beta+1,-\alpha-n \\
n-\nu+\beta+1
\end{array} ; \frac{1+y}{2}\right)= \\
& =(-1)^{n} \frac{\Gamma(n+\beta+1) \Gamma(n-\nu)}{2^{n-\nu} n ! \Gamma(n-\nu+\beta+1)}(1-y)^{n+\alpha-\nu}(1+y)^{n+\beta-\nu} F\left(\begin{array}{c}
-\nu, 2 n-\nu+\alpha+\beta+1 \\
n-\nu+\beta+1
\end{array} ; \frac{1+y}{2}\right)
\end{aligned}
$$

where $F$ is the Gauss hypergeometric function.

Similarly the integral for $J_{2}(y)$ can be computed. The result is:

$$
J_{2}(y)=(-1)^{n} \frac{2^{n+\alpha+\beta+1}}{(y+1)^{\nu+1}} \frac{\Gamma(n-\nu)}{\Gamma(-\nu) n !} \frac{\Gamma(n+\alpha+1) \Gamma(n+\beta+1)}{\Gamma(2 n+\alpha+\beta+2)} F\left(\begin{array}{c}
\nu+1, n+\beta+1 \\
2 n+\alpha+\beta+2
\end{array} ; \frac{2}{y+1}\right)
$$

Substitution of (22) and (23) in (19) and (20) and then (19) and (20) in (18) yields the following theorem, initially proven for $\operatorname{Re}(\nu)<0$, but by analytic continuation in $\nu$ valid for $\operatorname{Re}(\nu)<n$.

Theorem 4.3. For the approximate fractional Jacobi derivative, the following formula is valid:

$$
\begin{aligned}
W_{\delta, \alpha, \beta}^{\nu, n}[f](x) & =\frac{1}{\Gamma(-\nu)} \frac{1}{\delta^{\nu}} \int_{1}^{\infty} f(x+\delta y) \frac{1}{(y+1)^{\nu+1}} F\left(\begin{array}{c}
\nu+1, n+\beta+1 \\
2 n+\alpha+\beta+2
\end{array} ; \frac{2}{y+1}\right) d y+ \\
& +\frac{(-1)^{n} \Gamma(2 n+\alpha+\beta+2)}{2^{2 n-\nu+\alpha+\beta+1} \Gamma(n+\alpha+1) \Gamma(n-\nu+\beta+1)} \frac{1}{\delta^{\nu}} \\
& \int_{-1}^{1} f(x+\delta y)(1-y)^{n+\alpha-\nu}(1+y)^{n+\beta-\nu} F\left(\begin{array}{c}
-\nu, 2 n-\nu+\alpha+\beta+1 \\
n-\nu+\beta+1
\end{array} ; \frac{1+y}{2}\right) d y
\end{aligned}
$$

with $f(x)=O\left(\operatorname{Re}\left(x^{\nu-n-\varepsilon}\right)\right)$ as $x \rightarrow \infty$ and $\operatorname{Re}(\nu)<n$.

Remark 4.4. Note that the right-hand side of (24) is continuous in $\nu$ for $\operatorname{Re}(\nu) \leq n$. Thus, we can use (24) as a definition of $W_{\delta, \alpha, \beta}^{\nu, n}[f](x)$ for $\operatorname{Re}(\nu)=n$. We can also relax the condition for $f(x)$ as $x \rightarrow \infty$ to $f(x)=O\left(x^{-\varepsilon}\right)$, since the first integral on the right-hand side of (24) remains convergent then.

From this formula, one can see once more the validity of Proposition 4.1. For $\nu=0,1,2, \ldots, n$, the hypergeometric function in the first integral can be written as a Jacobi polynomial. The first term vanishes, because of the Gamma function $\Gamma(-\nu)=\Gamma(-n)$. In the second term, the hypergeometric function can be written as $P_{n}^{(\beta, \alpha)}(-y)$. Using (17) and taking the limit for $\delta \downarrow 0$, there remains the orthogonal derivative associated with the Jacobi polynomials:

$D^{n}[f](x)=\frac{\Gamma(2 n+\alpha+\beta+2) \Gamma(n+1)}{2^{n+\alpha+\beta+1} \Gamma(n+\alpha+1) \Gamma(n+\beta+1)} \lim _{\delta \downarrow 0} \frac{1}{\delta^{n}} \int_{-1}^{1} f(x+\delta y)(1-y)^{\alpha}(1+y)^{\beta} P_{n}^{(\alpha, \beta)}(y) d y$ which is exactly (7) for the case of the Jacobi polynomials.

For the special case that $\alpha \rightarrow \alpha-1 / 2$ and $\beta \rightarrow \alpha-1 / 2$, the Jacobi polynomials become the Gegenbauer polynomials. In that case, the hypergeometric functions in (24) can be expressed in terms 
of associated Legendre functions. For the hypergeometric function in the first term of (24) [13] (15.8.13 together with 14.3.7) gives:

$$
\frac{1}{(y+1)^{\nu+1}} F\left(\begin{array}{c}
\nu+1, n+\alpha+\frac{1}{2} \\
2 n+2 \alpha+1
\end{array} ; \frac{2}{y+1}\right)=(-1)^{n} \frac{2^{n+\alpha+1 / 2} \Gamma(n+\alpha+1)}{e^{i \pi(1 / 2-\alpha+\nu)} \sqrt{\pi} \Gamma(\nu+1)} \frac{Q_{n+\alpha-1 / 2}^{1 / 2-\alpha-n+\nu}(y)}{\left(\sqrt{y^{2}-1}\right)^{1 / 2-\alpha-n+\nu}}
$$

$Q_{\nu}^{\mu}(y)$ is the associated Legendre of the second kind. For the hypergeometric function in the second term of (24) [13] (15.8.1 together with 14.3.1) gives:

$$
F\left(\begin{array}{c}
-\nu, 2 n-\nu+2 \alpha \\
n-\nu+\alpha+\frac{1}{2}
\end{array} ; \frac{1-y}{2}\right)=\Gamma\left(n-\nu+\alpha+\frac{1}{2}\right) 2^{n+\alpha-\nu-1 / 2} \frac{\mathrm{P}_{n+\alpha-1 / 2}^{1 / 2-\alpha+n+\nu}(y)}{\left(\sqrt{1-y^{2}}\right)^{n+\alpha-\nu-1 / 2}}
$$

$\mathrm{P}_{\nu}^{\mu}(y)$ is the associated Legendre function of the first kind on the cut. Substitution gives for the approximate fractional Gegenbauer derivative with $\operatorname{Re}(\nu) \leq n$ :

$$
\begin{aligned}
W_{\delta, \alpha}^{\nu, n}[f](x)_{G} & =(-1)^{n} \frac{\Gamma(n+\alpha+1) 2^{n+\alpha-1 / 2}}{\sqrt{\pi}} \frac{1}{\delta^{\nu}} \int_{-1}^{1} f(x-\delta y) \frac{\mathrm{P}_{n+\alpha-1 / 2}^{1 / 2-\alpha-n+\nu}(y)}{\left(\sqrt{1-y^{2}}\right)^{1 / 2-\alpha-n+\nu}} d y- \\
& -(-1)^{n} \frac{e^{i \pi(\alpha-1 / 2)} 2^{n+\alpha+1 / 2} \Gamma(n+\alpha+1) \sin (\nu \pi)}{\pi \sqrt{\pi}} \frac{1}{\delta^{\nu}} \int_{1}^{\infty} f(x-\delta y) \frac{Q_{n+\alpha-1 / 2}^{1 / 2-\alpha-n+\nu}(y)}{\left(\sqrt{y^{2}-1}\right)^{1 / 2-\alpha-n+\nu}} d y
\end{aligned}
$$

Further specialization to $\alpha=1 / 2$ gives the approximate fractional Legendre derivative with $R e(\nu) \leq n$ :

$$
\begin{aligned}
W_{\delta, 1 / 2}^{\nu, n}[f](x)_{P} & =(-1)^{n} \frac{\Gamma(2 n+2)}{\Gamma(n+1) 2^{n+1}} \frac{1}{\delta^{\nu}} \int_{-1}^{1} f(x-\delta y) \frac{\mathrm{P}_{n}^{\nu-n}(y)}{\left(\sqrt{1-y^{2}}\right)^{\nu-n}} d y- \\
& -(-1)^{n} \frac{\sin (\nu \pi)}{\pi} \frac{\Gamma(2 n+2)}{\Gamma(n+1) 2^{n}} \frac{1}{\delta^{\nu}} \int_{1}^{\infty} f(x-\delta y) \frac{Q_{n}^{\nu-n}(y)}{\left(\sqrt{y^{2}-1}\right)^{\nu-n}} d y
\end{aligned}
$$

Remark 4.5. For the approximate fractional Gegenbauer derivative following the Riemann-Liouville definition, it can be shown that with $\operatorname{Re}(\nu) \leq n$ :

$$
\begin{aligned}
R_{\delta, \alpha}^{\nu, n}[f](x)_{G} & =\frac{2^{n+\alpha-1 / 2} \Gamma(n+\alpha+1)}{\sqrt{\pi}} \frac{1}{\delta^{\nu}} \int_{-1}^{1} f(x+\delta y) \frac{P_{n+\alpha-1 / 2}^{1 / 2-\alpha-n+\nu}(y)}{\left(\sqrt{1-y^{2}}\right)^{1 / 2-\alpha-n+\nu}} d y- \\
& -(-1)^{n} \frac{e^{i \pi(\alpha-1 / 2)} 2^{n+\alpha+1 / 2} \Gamma(n+\alpha+1) \sin (\nu \pi)}{\pi \sqrt{\pi}} \frac{1}{\delta^{\nu}} \int_{1}^{\infty} f(x+\delta y) \frac{Q_{n+\alpha-1 / 2}^{1 / 2-\alpha-n+\nu}(y)}{\left(\sqrt{y^{2}-1}\right)^{1 / 2-\alpha-n+\nu}} d y
\end{aligned}
$$

Remark 4.6. In the same way as we did in the Jacobi case, one can calculate the approximate fractional Laguerre derivative. Then, we start with (12). It follows:

$$
W_{\delta, \alpha}^{\nu, n}[f](x)_{L}=\frac{1}{\Gamma(n-\nu+\alpha+1)} \frac{1}{\delta^{\nu}} \int_{0}^{\infty} f(x+\delta y) y^{n-\nu+\alpha} e^{-y} M(-\nu ; n-\nu+\alpha+1 ; y) d y
$$

where $M$ is the confluent hypergeometric function and $f(x)=O\left(\operatorname{Re}\left(x^{\nu-n-\varepsilon}\right)\right)$ as $x \rightarrow \infty$ with $\operatorname{Re}(\nu) \leq n$. 


\section{The Frequency Response for the Approximate Fractional Orthogonal Derivative}

In Theorem 2.1, we gave the Fourier transform of the fractional derivative. It is possible to extend that theorem to the approximate fractional orthogonal derivative.

As in Theorem 2.1, let $F(\omega)$ and $G(\omega)$ be the Fourier transforms of $f$ and $g$, respectively. From (7), with $f$ absolutely integrable on $\mathbb{R}$ and $g=D_{\delta}^{n}[f]$, we immediately derive for the frequency response $H_{\delta}^{n}(\omega)$ :

$$
H_{\delta}^{n}(\omega)=\frac{G(\omega)}{F(\omega)}=\frac{k_{n} n !}{h_{n} \delta^{n}} \int_{\mathbb{R}} p_{n}(\xi) e^{-i \omega \delta \xi} d \mu(\xi)
$$

The exponential can be expanded as a power series. Because of the orthogonality property, it follows:

$$
\begin{aligned}
H_{\delta}^{n}(\omega) & =\frac{k_{n} n !}{h_{n} \delta^{n}} \int_{\mathbb{R}} p_{n}(\xi) \sum_{k=n}^{\infty} \frac{(-i \omega \delta \xi)^{k}}{k !} d \mu(\xi)= \\
& =\frac{k_{n} n !}{h_{n}}(-i \omega)^{n} \int_{\mathbb{R}} p_{n}(\xi) \xi^{n} \sum_{k=0}^{\infty} \frac{(-i \omega \delta \xi)^{k}}{(k+n) !} d \mu(\xi)
\end{aligned}
$$

We arrive at the following theorem:

Theorem 5.1. Let $F(\omega)$ and $G(\omega)$ be the Fourier transforms of the function $f$ and the function $g=W_{\delta}^{\nu, n}[f]$, respectively. Then (recall the convention after (5)):

$$
\begin{aligned}
H_{\delta}^{\nu}(\omega) & =\frac{k_{n} n !}{h_{n} \delta^{n}}(-1)^{n}(i \omega)^{\nu-n} \int_{\mathbb{R}} p_{n}(\xi) e^{-i \omega \delta \xi} d \mu(\xi)= \\
& =\frac{k_{n} n !}{h_{n}}(i \omega)^{\nu} \int_{\mathbb{R}} p_{n}(\xi) \xi^{n} \sum_{k=0}^{\infty} \frac{(-i \omega \delta \xi)^{k}}{(k+n) !} d \mu(\xi)
\end{aligned}
$$

Proof. Combine (26) and (27) with (9) and Theorem 2.1, and the result follows immediately.

Since $\int_{\mathbb{R}} p_{n}(\xi) e^{-i \omega \delta \xi} d \mu(\xi)$ is explicitly known for the classical orthogonal polynomials, we get explicit formulas for the frequency response in these cases. Furthermore, for the case of discrete Hahn polynomials, it is possible to compute the transfer function. We now treat two examples. First, we compute the frequency response in the case of the Jacobi polynomials. Then, we compute the frequency response in the case of the discrete Hahn polynomials.

\subsection{The Frequency Response for the Jacobi Polynomials}

For the Jacobi polynomials, the following integral is known [13] (18.17.16):

$$
\begin{aligned}
\int_{-1}^{1}(1-x)^{\alpha} & (1+x)^{\beta} P_{n}^{(\alpha, \beta)}(x) e^{i x y} d x= \\
& =\frac{(i y)^{n} e^{i y}}{n !} 2^{n+\alpha+\beta+1} B(n+\alpha+1, n+\beta+1) M(n+\alpha+1,2 n+\alpha+\beta+2 ;-2 i y)
\end{aligned}
$$

Using (15) and (16) and substitution of $y=-\omega \delta$ yields for (27) after some calculation:

$$
H_{\delta}^{\nu}(\omega)=(i \omega)^{\nu} e^{-i \omega \delta} M(n+\alpha+1,2 n+\alpha+\beta+2 ; 2 i \omega \delta)
$$


Using [13] (13.7.2) for $\omega \rightarrow \infty$, there remains:

$$
\left|H_{\delta}^{\nu}(\omega)\right| \sim \text { const } \cdot \omega^{\nu-n-\min (\alpha, \beta)}
$$

Hence, since $\nu<n$ :

$$
\lim _{\omega \rightarrow \infty}\left|H_{\delta}^{\nu}(\omega)\right|=0
$$

For the Gegenbauer polynomials, let $\alpha \rightarrow \alpha-\frac{1}{2}$ and $\beta \rightarrow \alpha-\frac{1}{2}$. Then:

$$
H_{\delta}^{\nu}(\omega)=(i \omega)^{\nu} e^{-i \omega \delta} M\left(n+\alpha+\frac{1}{2} ; 2 n+2 \alpha+1 ; 2 i \omega \delta\right)
$$

The confluent hypergeometric function can be transformed into a Bessel function. (13.6.9) gives:

$$
M\left(\nu+\frac{1}{2}, 2 \nu+1 ; 2 i z\right)=\Gamma(1+\nu) e^{i z}\left(\frac{2}{z}\right)^{\nu} J_{\nu}(z)
$$

Substitution in (29) gives:

$$
H_{\delta}^{\nu}(\omega)=(i \omega)^{\nu} \Gamma(n+\alpha+1)\left(\frac{2}{\omega \delta}\right)^{n+\alpha} J_{n+\alpha}(\omega \delta)
$$

The Bessel function can be written as a summation [13] (10.2.2). After substitution, there remains:

$$
H_{\delta}^{\nu}(\omega)=(i \omega)^{\nu} \Gamma(n+\alpha+1) \sum_{k=0}^{\infty} \frac{(-1)^{k}}{\Gamma(k+n+\alpha+1) k !}\left(\frac{\omega \delta}{2}\right)^{2 k}
$$

The right-hand side is real, except for the factor $(i \omega)^{\nu}$. The variable $n$ is equal to the next integer greater then $\nu$. For a certain value of $\nu$ with $n-1 \leq \nu \leq n$, the summation is independent of $\nu$. For the modulus of the transfer function, we obtain:

$$
\left|H_{\delta}^{\nu}(\omega)\right|=\omega^{\nu} \Gamma(n+\alpha+1)\left|\sum_{k=0}^{\infty} \frac{(-1)^{k}}{\Gamma(k+n+\alpha+1) k !}\left(\frac{\omega \delta}{2}\right)^{2 k}\right|
$$

$\alpha$ is a free parameter. For $\alpha=\frac{1}{2}$, we get the frequency response of the approximate fractional Legendre derivative. Then, (31) becomes:

$$
\left|H_{\delta}^{\nu}(\omega)\right|=\omega^{\nu} \frac{\Gamma(2 n+2)}{2^{n} \Gamma(n+1)} \frac{1}{(\omega \delta)^{n}}\left|j_{n}(\omega \delta)\right|
$$

where $j_{n}$ is a spherical Bessel function [13] (10.49.2). Special cases of $j_{n}$ are:

$$
\begin{gathered}
j_{0}(z)=\frac{1}{z} \sin z \\
j_{1}(z)=\frac{1}{z^{2}}(\sin z-z \cos z) \\
j_{2}(z)=\frac{1}{z^{3}}\left(\left(3-z^{2}\right) \sin z-3 z \cos z\right) \\
j_{3}(z)=\frac{1}{z^{4}}\left(\left(15-6 z^{2}\right) \sin z-\left(15-z^{2}\right) z \cos z\right)
\end{gathered}
$$

The above formulas remain valid for $\nu=n$, where we get the transfer functions as in [8] (Section 5). 
Remark 5.2. In [11], we apply this theory to a fractional differentiating filter.

Remark 5.3. From Theorem 5.1, we get:

$$
\frac{1}{\sqrt{2 \pi}} \int_{\mathbb{R}} W_{\delta}^{\nu, n}[f](x) e^{i \omega x} d x=H_{\delta}^{\nu}(\omega) F(\omega)
$$

with $H_{\delta}^{\nu}(\omega)$ explicitly and quickly computable in special cases, for instance the Jacobi case. Thus, if:

$$
H_{\delta}^{\nu}(\omega)=\frac{1}{\sqrt{2 \pi}} \frac{1}{\delta^{\nu}} \int_{\mathbb{R}} h_{\delta}^{\nu}(x) e^{i \sigma \omega x} d x
$$

for some function h, then after the inverse Fourier transform, there remains the convolution integral:

$$
W_{\delta}^{\nu, n}[f](x)=\frac{1}{\sqrt{2 \pi}} \frac{1}{\delta^{\nu}} \int_{\mathbb{R}} f(x-\delta y) h_{\delta}^{\nu}(y) d y
$$

If we compare, in the case of a finite orthogonality, this last formula with (11), then we can read off $h$ from (11). In particular, in the Jacobi case, we can read off $h$ from (24). This gives:

$$
h_{\delta}^{\nu}(y)= \begin{cases}\frac{\sqrt{2 \pi}}{\Gamma(-\nu) \delta^{\nu+1}} \frac{1}{(1+y)^{\nu+1}} F\left(\begin{array}{c}
\nu+1, n+\beta+1 \\
2 n+\alpha+\beta+2
\end{array} ; \frac{2}{1+y}\right) & y>1 \\
\frac{\sqrt{2 \pi} \Gamma(2 n+\alpha+\beta+2)}{2^{2 n-\nu+\alpha+\beta+1} \Gamma(n+\alpha+1) \Gamma(n-\nu+\beta+1) \delta^{\nu+1}} & -1<y<1 \\
(1+y)^{n+\beta-\nu}(1-y)^{n+\alpha-\nu} F\left(\begin{array}{cc}
-\nu, 2 n-\nu+\alpha+\beta+1 \\
n-\nu+\beta+1
\end{array} ; \frac{1+y}{2}\right) & y<-1\end{cases}
$$

Its Fourier transform $H_{\delta}^{\nu}(\omega)$ is given by (29).

\subsection{The Frequency Response for the Hahn Polynomials}

We start with formula (27) for the approximate fractional orthogonal derivative and apply this formula for the discrete case with weights $w(x)$ on points $x \in\{0,1, \ldots, N\}$. This gives:

$$
H_{\delta}^{\nu}(\omega)=\frac{k_{n} n !}{h_{n} \delta^{n}}(-1)^{n}(i \omega)^{\nu-n} \sum_{x=0}^{N} p_{n}(x) w(x) e^{-i x \delta \omega}
$$

When applying the summation to the discrete Hahn polynomials, we get:

$$
S=\sum_{x=0}^{N} p_{n}(x) w(x) e^{-i x \delta \omega}=\sum_{x=0}^{N} Q_{n}(x ; \alpha, \beta, N) w(x) e^{-i \delta \omega x}
$$

For the Hahn polynomials, we use [14] (9.5.10):

$$
Q_{n}(x ; \alpha, \beta, N) w(x)=\frac{(-1)^{n}(\beta+1)_{n}}{(-N)_{n}} \nabla_{x}^{n}\left(\frac{(\alpha+n+1)_{x}}{x !} \frac{(\beta+n+1)_{N-n-x}}{(N-n-x) !}\right)
$$

where:

$$
\nabla_{x}(f(x)):=f(x)-f(x-1)
$$


and get:

$$
S=\frac{(-1)^{n}(\beta+1)_{n}}{(-N)_{n}} \sum_{x=0}^{N} e^{-i \delta \omega x} \nabla^{n}\left(\frac{(\alpha+n+1)_{x}}{x !} \frac{(\beta+n+1)_{N-n-x}}{(N-n-x) !}\right)
$$

To compute this sum, we use partial summation. The general formula for partial summation is:

$$
\sum_{j=0}^{N}(\nabla f)(j) g(j)=f(N) g(N)-f(-1) g(0)-\sum_{j=0}^{N-1} f(j)(\nabla g)(j+1)
$$

In particular, if $f(N)=f(-1)=0$, then:

$$
\sum_{j=0}^{N}(\nabla f)(j) g(j)=-\sum_{j=0}^{N-1} f(j)(\nabla g)(j+1)
$$

Applying this formula $n$ times and remarking that $f(N-n)=f(-1)=0$ gives:

$$
\sum_{j=0}^{N}(\nabla f)^{n}(j) g(j)=(-1)^{n} \sum_{j=0}^{N-n} f(j)(\nabla g)^{n}(j+n)
$$

Applying this formula to (34) gives:

$$
S=\frac{(\beta+1)_{n}}{(-N)_{n}} \sum_{x=0}^{N-n} \nabla^{n}\left(e^{-i \delta \omega(x+n)}\right) \frac{(\alpha+n+1)_{x}}{x !} \frac{(\beta+n+1)_{N-n-x}}{(N-n-x) !}
$$

The iterated differences of the exponential are known. This gives:

$$
\begin{aligned}
S & =\frac{(\beta+1)_{n}}{(-N)_{n}}\left(e^{-i \delta \omega}-1\right)^{n} \sum_{x=0}^{N-n} e^{-i \delta \omega x} \frac{(\alpha+n+1)_{x}}{x !} \frac{(\beta+n+1)_{N-n-x}}{(N-n-x) !}= \\
& =\frac{(\beta+1)_{n}}{(-N)_{n}}\left(e^{-i \delta \omega}-1\right)^{n} \sum_{x=0}^{N-n} \frac{(\alpha+n+1)_{x}(\beta+n+1)_{N-n}}{(-\beta-N)_{x}} \frac{(-N+n)_{x}}{(N-n) !} \frac{\left(e^{-i \delta \omega}\right)^{x}}{x !}= \\
& =\frac{(\beta+1)_{n}(\beta+n+1)_{N-n}}{(-N)_{n}(N-n) !}\left(e^{-i \delta \omega}-1\right)^{n} \sum_{x=0}^{N-n} \frac{(\alpha+n+1)_{x}(-N+n)_{x}}{(-\beta-N)_{x}} \frac{\left(e^{-i \delta \omega}\right)^{x}}{x !}= \\
& =\frac{(\beta+1)_{N}}{N !}\left(1-e^{-i \delta \omega}\right)^{n} F\left(\begin{array}{c}
-N+n, \alpha+n+1 \\
-\beta-N
\end{array}\right)
\end{aligned}
$$

Then, for the frequency response, there remains:

$$
H_{\delta}^{\nu}(\omega)=\frac{k_{n} n !}{h_{n} \delta^{n}}(-1)^{n}(i \omega)^{\nu-n} \frac{(\beta+1)_{N}}{N !}\left(1-e^{-i \delta \omega}\right)^{n} F\left(\begin{array}{c}
-N+n, \alpha+n+1 \\
-\beta-N
\end{array} ; e^{-i \delta \omega}\right)
$$

For the Hahn polynomials, Tables 18.19 .1 and 18.19.2 in [13] give:

$$
\frac{k_{n}}{h_{n}}=(-1)^{n} \frac{(2 n+\alpha+\beta+1)}{(\beta+1)_{n}} \frac{(n+\alpha+\beta+1)_{n}}{(n+\alpha+\beta+1)_{N+1}} \frac{N !}{n !}
$$

Substitution in (36) gives:

$$
H_{\delta}^{\nu}(\omega)=(i \omega)^{\nu-n} \frac{\left(1-e^{-i \delta \omega}\right)^{n}}{\delta^{n}} \frac{\Gamma(N+\beta+1) \Gamma(2 n+\alpha+\beta+2)}{\Gamma n+\beta+1) \Gamma(N+n+\alpha+\beta+2)} F\left(\begin{array}{c}
-N+n, \alpha+n+1 \\
-\beta-N
\end{array} ; e^{-i \delta \omega}\right)
$$


Remark 5.4. From the summation (33) together with (35), one can derive the integral formula when letting $N \rightarrow \infty$. For this purpose, we apply [13] (Table 18.19, (15.8.7) and (18.20.5)) to (35). With $\delta=-1$, this gives:

$$
\begin{aligned}
& \sum_{x=0}^{N}{ }_{3} F_{2}\left(\begin{array}{c}
-n, n+\alpha+\beta+1,-x \\
\alpha+1,-N
\end{array} ; 1\right) \frac{(\alpha+1)_{x}(\beta+1)_{N-x}}{x !(N-x) !} e^{i \omega x}= \\
& =\frac{(\beta+1)_{n}(2 n+\alpha+\beta+2)_{N-n}}{N !}\left(1-e^{i \omega}\right)^{n} F\left(\begin{array}{c}
-N+n, \alpha+n+1 \\
2 n+\alpha+\beta+2
\end{array} ; 1-e^{i \omega}\right)
\end{aligned}
$$

The summation can also be done over the set $x \in \frac{1}{N}\{0,1, \ldots, N\}$. The $N+1$ points then lie in the interval $[0,1]$. This gives:

$$
\begin{aligned}
& \sum_{x=0,1 / N, \ldots 1}{ }_{3} F_{2}\left(\begin{array}{c}
-n, n+\alpha+\beta+1,-N x \\
\alpha+1,-N
\end{array} ; 1\right) \frac{(\alpha+1)_{N x}(\beta+1)_{N(1-x)}}{(N x) !(N-N x) !} e^{i \omega x}= \\
& =\frac{(\beta+1)_{n}(2 n+\alpha+\beta+2)_{N-n}}{N !}\left(1-e^{i \omega / N}\right)^{n} F\left(\begin{array}{c}
-N+n, \alpha+n+1 \\
2 n+\alpha+\beta+2
\end{array} ; 1-e^{i \omega / N}\right)
\end{aligned}
$$

This formula can be written as:

$$
\begin{gathered}
\frac{\Gamma(\alpha+1) \Gamma(\beta+1)}{N^{\alpha+\beta+1}} \sum_{x=0,1 / N, . .1}{ }_{3} F_{2}\left(\begin{array}{c}
-n, n+\alpha+\beta+1,-N x \\
\alpha+1,-N
\end{array} ; 1\right) \frac{(\alpha+1)_{N x}(\beta+1)_{N(1-x)}}{(N x) !(N-N x) !} e^{i \omega x}= \\
=\frac{\Gamma(\alpha+1) \Gamma(\beta+1)}{N^{n+\alpha+\beta+1}} \frac{(\beta+1)_{n}(2 n+\alpha+\beta+2)_{N-n}}{N !}\left(N\left(1-e^{i \omega / N}\right)\right)^{n} \\
F\left(\begin{array}{c}
-N+n, \alpha+n+1 \\
2 n+\alpha+\beta+2
\end{array} ; \frac{N\left(e^{i \omega / N}-1\right)}{-N}\right)
\end{gathered}
$$

Taking the limit for $N \rightarrow \infty$ and using [13] (5.11.12) gives formally:

$$
\begin{aligned}
\int_{0}^{1} F\left(\begin{array}{c}
-n, n+\alpha+\beta+1 \\
\alpha+1
\end{array}\right. & ; x) x^{\alpha}(1-x)^{\beta} e^{i \omega x} d x= \\
& =\frac{\Gamma(\alpha+1) \Gamma(n+\beta+1)}{\Gamma(2 n+\alpha+\beta+2)}(-i \omega)^{n} M(n+\alpha+1 ; 2 n+\alpha+\beta+2 ; i \omega)
\end{aligned}
$$

This is the same formula as (29) after some transformation.

Remark 5.5. In [15], the authors compute the fractional derivative using the Fourier transform. They also design a fractional digital filter. They demonstrate the filter performance by using a block signal as the input. In our opinion, this demonstration is less convincing, because by absence of an integrating factor, the high frequencies are not suppressed and because the plots are not given as log-log plots. One should compare with our approach in [11] (Section 6) where we give a log-log plot of the modulus of the frequency response. 


\section{Deriving a Formula for a Fractional Derivative Starting with a Suitable Frequency Response}

For the second expression in (28) of the frequency response of the approximate fractional orthogonal derivative, we see that:

$$
H_{\delta}^{\nu}(\omega)=(i \omega+0)^{\nu}(1+O(\delta \omega))=\frac{1}{\delta^{\nu}}(i \delta \omega+0)^{\nu}(1+O(\delta \omega))
$$

as $\delta \omega \downarrow 0$. Conversely, if we have some explicit function $H_{\delta}^{\nu}(\omega)$ behaving like $(i \omega)^{\nu}(1+O(\delta \omega))$ as $\delta \omega \downarrow 0$, of which the inverse Fourier transform $h(x)$ exists and is explicitly known, then the convolution product of $f$ with $h$ gives a formula for an approximate fractional derivative. The next derivation demonstrates this method.

Set

$$
F(\omega)=\frac{1}{\sqrt{2 \pi}} \int_{-\infty}^{\infty} e^{i \omega x} f(x) d x
$$

When taking the inverse Fourier transform of (5), it follows for the Weyl derivative:

$$
W^{\nu}[f](x)=\frac{1}{\sqrt{2 \pi}} \int_{-\infty}^{\infty} e^{-i \omega x}(i \omega+0)^{\nu} F(\omega) d \omega
$$

Now, we look for a function with the following property:

$$
\lim _{\delta \downarrow 0} H_{\delta}^{\nu}(i \omega+0)=(i \omega+0)^{\nu}
$$

Substitution in (37) with $W^{\nu}[f](x)=\lim _{\delta \downarrow 0} W_{\delta}^{\nu}[f](x)$ yields (care should be taken with the interchanging of the limit and the integral):

$$
W_{\delta}^{\nu}[f](x)=\frac{1}{\sqrt{2 \pi}} \int_{-\infty}^{\infty} e^{-i \omega x} H_{\delta}^{\nu}(i \omega+0) F(\omega) d \omega
$$

Suppose $H_{\delta}^{\nu}$ can be written as a Fourier transform:

$$
H_{\delta}^{\nu}(i \omega+0)=\frac{1}{\delta^{\nu}} \frac{1}{\sqrt{2 \pi}} \int_{-\infty}^{\infty} h_{\delta}^{\nu}(y) e^{i \delta \omega y} d y
$$

Then, for the approximate Weyl derivative, the convolution integral follows:

$$
W_{\delta}^{\nu}[f](x)=\frac{1}{\sqrt{2 \pi}} \frac{1}{\delta^{\nu}} \int_{-\infty}^{\infty} h_{\delta}^{\nu}(y) f(x-\delta y) d y
$$

Taking the limit for $\delta \downarrow 0$ yields:

$$
\frac{d^{\nu}}{d x^{\nu}} f(x)=\lim _{\delta \rightarrow 0} W_{\delta}^{\nu}[f](x)=\frac{1}{\sqrt{2 \pi}} \lim _{\delta \rightarrow 0} \frac{1}{\delta^{\nu}} \int_{-\infty}^{\infty} h_{\delta}^{\nu}(y) f(x-\delta y)
$$

Originally, the author started his work on the fractional derivative with the special case $H(\omega)=\omega^{-\beta} J_{\alpha}(\omega)$. Taking the inverse Fourier transform, he derived the formula for the fractional Gegenbauer derivative (25) with two free parameters. No use was made of the orthogonality property of the Gegenbauer polynomials. 
Because the Bessel function is a special case of the confluent hypergeometric function, the next extension arises when using the inverse Fourier transform of the function $H(\omega)=\omega^{b-1} M(a, c ; i k \omega)$. Then, there are three free parameters. By (30), the approximate fractional Jacobi derivative will arise. A possible next extension is the use of the generalized confluent hypergeometric function. The frequency response can be written as $H(\omega)=\omega^{\gamma-1}{ }_{A} F_{A}[(a),(b) ; i k \omega]$. There are $2 A+1$ free parameters. Possibly, extensions could involve the Meijer $G$-function and the Fox $H$-function. See [16].

The main properties of the function $H(\omega)$ are that its inverse Fourier transform exists and $H_{\delta}^{\nu}(\omega)=(i \omega+0)^{\nu}(1+O(\delta \omega))$ as $\delta \omega \downarrow 0$.

As an example of the method, we derive the formula for the fractional derivative using the inverse Fourier transform of the confluent hypergeometric function $H(\omega)=(i \omega+0)^{b-1} M(a ; c ; i \omega)$. For this transform, it can be derived (see Appendix A):

$$
\begin{aligned}
& F^{-1}\left[(i \omega+0)^{b-1} M(a, c ; i \omega)\right](y)= \\
& = \begin{cases}0 & 1<y, \\
\frac{\sqrt{2 \pi} \Gamma(c)}{\Gamma(a) \Gamma(1-a-b+c)} y^{a-b}(1-y)^{c-a-b} F\left(\begin{array}{c}
1-b, c-b \\
1-a-b+c
\end{array} ; 1-y\right) & 0<y<1, \\
\frac{\sqrt{2 \pi}}{\Gamma(1-b)}(1-y)^{-b} F\left(\begin{array}{cc}
b, c-a \\
c
\end{array} \frac{1}{1-y}\right) & y<0 .\end{cases}
\end{aligned}
$$

with conditions for the parameters: $0<b<\min (a, c-a)$. Application of (39) with $\nu=b-1$ gives:

$$
\begin{aligned}
& \frac{d^{\nu}}{d x^{\nu}} f(x)=\lim _{\delta \rightarrow 0}\left(\frac { 1 } { \Gamma ( - \nu ) } \frac { 1 } { \delta ^ { \nu } } \int _ { - \infty } ^ { 0 } \frac { 1 } { ( 1 - y ) ^ { \nu + 1 } } F \left(\begin{array}{c}
\left.\nu+1, c-a ; \frac{1}{1-y}\right) f(x-\delta y) d y+ \\
c
\end{array}\right.\right. \\
& \left.\quad+\frac{\Gamma(c)}{\Gamma(a) \Gamma(c-a-\nu)} \frac{1}{\delta^{\nu}} \int_{0}^{1} y^{a-\nu-1}(1-y)^{c-a-\nu-1} F\left(\begin{array}{c}
-\nu, c-\nu-1 \\
c-a-\nu
\end{array} ; 1-y\right) f(x-\delta y) d y\right)
\end{aligned}
$$

Because the fractional derivative should be calculated, we set $\nu>0$. This gives $b>1$. In (41), there are two free parameters, namely $a$ and $c$. For the conditions, we find: $0<\nu<\min (a, c-a)-1$.

Replacing in both integrals $y$ by $\frac{1-y}{2}$ and $\delta$ by $2 \delta$ gives:

$$
\begin{aligned}
& \frac{d^{\nu}}{d x^{\nu}} f(x)=\lim _{\delta \rightarrow 0}\left(\frac{1}{\Gamma(-\nu)} \frac{1}{\delta^{\nu}} \int_{1}^{\infty} \frac{1}{(1+y)^{\nu+1}} F\left(\begin{array}{c}
\nu+1, c-a \\
c
\end{array} ; \frac{2}{1+y}\right) f(x+\delta y) d y+\right. \\
& \left.+\frac{\Gamma(c)}{2^{c-\nu-1} \Gamma(a) \Gamma(c-a-\nu)} \frac{1}{\delta^{\nu}} \int_{-1}^{1}(1-y)^{a-\nu-1}(1+y)^{c-a-\nu-1} F\left(\begin{array}{c}
-\nu, c-\nu-1 \\
c-a-\nu
\end{array} ; \frac{1+y}{2}\right) f(x+\delta y) d y\right)
\end{aligned}
$$

For $a=n+\alpha+1$ and $c=2 n+\alpha+\beta+2$, this formula is exactly the formula of the fractional Jacobi derivative (4.13) by taking the limit for $\delta \rightarrow 0$.

Remark 6.1. For the first method, we use consecutive (9) and (11), which lead to (24) and to (29). As a side result of this first method, we get (29) as the Fourier transform of (32); see Remark 5.2. The resulting inverse Fourier transform coincides after appropriate substitutions with (40). 
Remark 6.2. In the second method, no use is made of the orthogonality property. Therefore, for extending this method with, for example, the generalized confluent hypergeometric function, one cannot see directly what functions should be used with the first method to obtain the same result. Then, there remains the question if, in that case, there are some orthogonal polynomials (maybe yet unknown) that fulfill the first method.

Remark 6.3. One of the properties of the orthogonal derivative is that for high frequencies, the modulus of the frequency response tends to zero. Our example satisfies this condition.

Remark 6.4. From (42), the next theorem follows, which is proven in Appendix B.

Theorem 6.5. Let $k+\nu<a<c-\nu$ and $0<k<\nu<a+1$. Then:

$$
\begin{aligned}
& \frac{1}{\Gamma(-\nu)} \int_{1}^{\infty} y^{k} \frac{1}{(1+y)^{\nu+1}} F\left(\begin{array}{c}
\nu+1, c-a \\
c
\end{array} ; \frac{2}{1+y}\right) d y+ \\
+ & \frac{\Gamma(c)}{2^{c-\nu-1} \Gamma(a) \Gamma(c-a-\nu)} \int_{-1}^{1}(-y)^{k}(1+y)^{a-\nu-1}(1-y)^{c-a-\nu-1} F\left(\begin{array}{c}
-\nu, c-\nu-1 \\
c-a-\nu
\end{array} ; \frac{1-y}{2}\right) d y=0
\end{aligned}
$$

\section{The Fractional Derivative Arising from Some Particular Functions}

In this section, a demonstration is given of the second method for derivation of a formula of the fractional derivative based on some special functions. Both examples use an inverse Fourier transform in order to calculate a formula for the fractional derivative. The results can be used as definitions of a two-sided fractional derivative.

\section{Example 1:}

Consider the function:

$$
H_{a}^{\nu}(\omega)=(i \omega+0)^{\nu} e^{-a|\omega|} \quad \nu>-1 \quad a>0
$$

Clearly, if $a \downarrow 0$, then $H(\omega) \rightarrow(i \omega+0)^{\nu}$. We calculate the inverse Fourier transform:

$$
\begin{aligned}
h_{a}^{\nu}(y) & =\frac{1}{\sqrt{2 \pi}} \int_{-\infty}^{\infty}(i \omega+0)^{\nu} e^{-a|\omega|} e^{-i \omega y} d \omega= \\
& =\frac{e^{i \pi \nu / 2}}{\sqrt{2 \pi}} \int_{0}^{\infty} \omega^{\nu} e^{-a \omega} e^{-i \omega y} d \omega+\frac{e^{-i \pi \nu / 2}}{\sqrt{2 \pi}} \int_{0}^{\infty} \omega^{\nu} e^{-a \omega} e^{i \omega y} d \omega= \\
& =\frac{\Gamma(\nu+1) e^{i \pi / 2}}{\sqrt{2 \pi}}\left[\frac{1}{(y+i a)^{\nu+1}}-\frac{1}{(y-i a)^{\nu+1}}\right]
\end{aligned}
$$

Hence, with:

$$
F(x)=\frac{1}{\sqrt{2 \pi}} \int_{-\infty}^{\infty} f(\omega) e^{i \omega x} d \omega
$$

we obtain:

$$
\begin{aligned}
\frac{d^{\nu}}{d x^{\nu}} f(x) & =\frac{1}{\sqrt{2 \pi}} \lim _{a \rightarrow 0} \int_{-\infty}^{\infty} F(\omega)(i \omega+0)^{\nu} e^{-a|\omega|} e^{-i \omega y} d \omega= \\
& =\frac{\Gamma(\nu+1)}{2 \pi i} \lim _{a \rightarrow 0} \int_{-\infty}^{\infty}\left[\frac{1}{(y-i a)^{\nu+1}}-\frac{1}{(y+i a)^{\nu+1}}\right] f(x+y) d y
\end{aligned}
$$


Care should be taken when $a \downarrow 0$. For $-\infty<y \leq 0$, there is a cut, so we have to use a well-chosen contour to calculate the integral. In that case, there remains:

$$
R^{\nu}[f](x)=\frac{1}{\sqrt{2 \pi}} \int_{-\infty}^{\infty} F(\omega)(i \omega+0)^{\nu} e^{-i \omega y} d \omega=\frac{\Gamma(\nu+1)}{2 \pi i} \int_{C} f(x+y) \frac{1}{y^{\nu+1}} d y
$$

Here, $C$ is the contour with:

$$
C=\{y-i 0 \mid-\infty<y<-r\} \cup\{z \in \mathbb{C}|| z \mid=r\} \cup\{y+i 0 \mid-r>y>-\infty\}
$$

This formula is well known in the literature. See, for example, $[4,17,18]$. If $\nu<0$, then there are no convergence problems.

After application of [13] (15.4.9), there remains:

$$
\frac{d^{\nu}}{d x^{\nu}} f(x)=\frac{\Gamma(\nu+2)}{\pi} \lim _{a \rightarrow 0} \frac{1}{a^{\nu-1}} \int_{-\infty}^{\infty} \frac{1}{y^{\nu+2}} F\left(\begin{array}{c}
\frac{\nu+2}{2}, \frac{\nu+2}{2}+\frac{1}{2} \\
\frac{3}{2}
\end{array}-\frac{a^{2}}{y^{2}}\right) f(x+y) d y
$$

The quadratic argument in the hypergeometric function suggests a connection with the pseudo Jacobi polynomials. They are orthogonal with the orthogonality property [14] (9.9.2).

$$
\int_{-\infty}^{\infty} P_{m}(x ; 0, N) P_{n}(x ; 0, N)\left(1+x^{2}\right)^{-N-1} d x=0 \quad n \neq m, \quad n, m=0,1, \ldots, N
$$

[14] (9.9.2) gives:

$$
P_{2 n}(x ; 0, N)=(-2 i)^{2 n}(-N)_{2 n}(2 n-2 N-1)_{2 n} F\left(\begin{array}{c}
-2 n, 2 n-2 N-1 \\
-N
\end{array} ; \frac{1-i x}{2}\right)
$$

With [13] (15.8.18 and 15.8.7), there remains:

$$
P_{2 n}(x ; 0,2 n)=\frac{(-1)^{n}}{2 n+1} F\left(\begin{array}{c}
-n,-n-\frac{1}{2} ;-x^{2} \\
\frac{1}{2}
\end{array}\right)
$$

and with [13] (15.4.7), there remains with a scaling factor $a$ :

$$
\begin{aligned}
P_{2 n}\left(\frac{x}{a} ; 0,2 n\right) & =\frac{(-1)^{n}}{2(2 n+1)}\left((1+i a x)^{2 n+1}-(1-i a x)^{2 n+1}\right)= \\
& =\frac{1}{2(2 n+1) i a}\left(a^{2}+x^{2}\right)^{2 n+1}\left(\frac{1}{(x-i a)^{2 n+1}}-\frac{1}{(x+i a)^{2 n+1}}\right)
\end{aligned}
$$

Substituting $\nu=2 n$ in (43) gives:

$$
\frac{d^{2 n}}{d x^{2 n}} f(x)=\frac{\Gamma(2 n+2)}{\pi} \lim _{a \rightarrow 0} \frac{1}{a^{4 n+1}} \int_{-\infty}^{\infty} \frac{P_{2 n}(y ; 0,2 n)}{\left(1+y^{2}\right)^{2 n+1}} f(x+a y) d z
$$

Thus, we arrive at a special case of (13) associated with the pseudo Jacobi polynomials.

When substituting $z=\frac{y}{i a}$ in the integral (44), the hypergeometric function can be written as an associated Legendre function of the second kind. This gives:

$$
\frac{d^{\nu}}{d x^{\nu}} f(x)=\frac{e^{-(\nu+1 / 2) \pi i} a}{\pi} \int_{-\infty}^{\infty} \frac{Q_{0}^{\nu+1}(z)}{\left(\sqrt{z^{2}-1}\right)^{\nu+1}} f(x+i a z) d z
$$




\section{Example 2:}

Consider the function:

$$
H^{\nu}(\omega)=(i \omega+0)^{\nu} e^{-\omega^{2} / 2}
$$

The inverse Fourier transform is known [19] (121(23) (note the factor $\sqrt{2 \pi}$ ):

$$
h^{\nu}(y)=e^{-y^{2} / 4} D_{\nu}(y) \quad \operatorname{Re}(\nu)>-1
$$

Here, $D_{\nu}(y)$ is a parabolic cylinder function [20] (8.2(2)). With the known method, one can derive for the fractional derivative:

$$
\frac{d^{\nu}}{d x^{\nu}} f(x)=\lim _{\delta \rightarrow 0} \frac{1}{\delta^{\nu}} \int_{-\infty}^{\infty} e^{-y^{2} / 4} D_{\nu}(y) f(x-\delta y) d y
$$

(46) can also be computed when (13) will be applied to the Hermite polynomials. It follows:

$$
W_{\delta}^{\nu, n}[f](x)=\frac{(-1)^{n}}{\sqrt{2 \pi} \Gamma(n-\nu)} \frac{1}{\delta^{\nu}} \int_{-\infty}^{\infty} f(x+\delta y)\left[\int_{-\infty}^{y}(y-u)^{n-\nu-1} H_{n}(u) e^{-u^{2}} d u\right] d y
$$

For the integral between the square brackets, we use the Rodrigues formula. It follows:

$$
I=\int_{-\infty}^{y}(y-u)^{n-\nu-1} H_{n}(u) e^{-u^{2}} d u=(-1)^{n} \int_{-\infty}^{y}(y-u)^{n-\nu-1} \frac{d^{n}}{d u^{n}}\left[e^{-u^{2}}\right] d u
$$

Repeated partial integration $n$ times gives:

$$
I=(-1)^{n} \frac{\Gamma(n-\nu)}{\Gamma(-\nu)} \int_{-\infty}^{y}(y-u)^{-\nu-1} e^{-u^{2}} d u=(-1)^{n} \frac{\Gamma(n-\nu)}{\Gamma(-\nu)} e^{-y^{2}} \int_{0}^{\infty} u^{-1-\nu} e^{-u^{2}+2 y u} d u
$$

This integral is well known [19] (I.313(13)):

$$
\int_{0}^{\infty} u^{-\nu-1} e^{-u^{2}+2 y u} d u=2^{\nu / 2} \Gamma(-\nu) e^{y^{2} / 2} D_{\nu}(-y \sqrt{2})
$$

Substitution gives:

$$
W_{\delta}^{\nu, n}[f](x)=\frac{1}{\sqrt{2 \pi}} \frac{2^{\nu / 2}}{\delta^{\nu}} \int_{-\infty}^{\infty} e^{-y^{2} / 2} D_{\nu}(-y \sqrt{2}) f(x+\delta y) d y
$$

Replacing $y$ by $-\frac{y}{\sqrt{2}}$ and $\delta$ by $\delta \sqrt{2}$ gives:

$$
W_{\delta}^{\nu, n}[f](x)=\frac{1}{\sqrt{2 \pi}} \frac{1}{\delta^{\nu}} \int_{-\infty}^{\infty} e^{-y^{2} / 4} D_{\nu}(y) f(x-\delta y) d y
$$

This result is the same as (44) when taking the limit. When $\nu$ is an integer $n$, we obtain:

$$
D_{n}(y)=2^{-n / 2} e^{-y^{2} / 4} H_{n}\left(\frac{y}{\sqrt{2}}\right)
$$

Substitution in (47) gives:

$$
\frac{d^{n}}{d x^{n}} f(x)=\frac{2^{-n / 2}}{\sqrt{2 \pi}} \lim _{\delta \rightarrow 0} \frac{1}{\delta^{n}} \int_{-\infty}^{\infty} e^{-y^{2} / 2} H_{n}\left(\frac{y}{\sqrt{2}}\right) f(x-\delta y) d y
$$


Replacing $y$ by $y \sqrt{2}$ and $\delta$ by $\frac{\delta}{\sqrt{2}}$, there arises the formula for the Hermite derivative:

$$
\frac{d^{n}}{d x^{n}} f(x)=\frac{1}{\sqrt{\pi}} \lim _{\delta \rightarrow 0} \frac{1}{\delta^{n}} \int_{-\infty}^{\infty} e^{-y^{2} / 2} H_{n}(y) f(x-\delta y) d y
$$

\section{Acknowledgments}

I thank T.H. Koornwinder for his help and time offered during my writing of this paper. Without his very stimulating enthusiasm and everlasting patience, this work could not have been done.

\section{A. Appendix: The Inverse Fourier Transform of the Function $F(\omega)=(i \omega+0)^{b-1} M(a, c ; i \omega)$}

In this section, we prove:

$$
\begin{aligned}
F^{-1} & {\left[(i \omega+0)^{b-1} M(a, c ; i \omega)\right](y)=} \\
& = \begin{cases}0 & 1<y \\
\sqrt{2 \pi} \frac{\Gamma(c)}{\Gamma(a) \Gamma(1-a-b+c)} y^{a-b}(1-y)^{c-a-b} F\left(\begin{array}{c}
1-b, c-b \\
c+1-a-b
\end{array} ; 1-y\right) & 0<y<1 \\
\frac{\sqrt{2 \pi}}{\Gamma(1-b)} \frac{1}{(1-y)^{b}} F\left(\begin{array}{cc}
b, c-a \\
c
\end{array} \frac{1}{1-y}\right) & y<0\end{cases}
\end{aligned}
$$

with conditions for the parameters: $a, b, c \in \mathbb{R}$ and $0<b<\min (a, c-a)$.

The inverse Fourier transform of the function $M(a, c ; i \omega)](y)$ is given by:

$$
\begin{aligned}
I & =F^{-1}\left[(i \omega+0)^{b-1} M(a, c ; i \omega)\right](y)=\frac{1}{\sqrt{2 \pi}} \int_{-\infty}^{\infty}(i \omega+0)^{b-1} M(a, c ; i \omega) e^{-i \omega y} d \omega= \\
& =\frac{e^{i \pi(b-1) / 2}}{\sqrt{2 \pi}} \int_{0}^{\infty} \omega^{b-1} M(a, c ; i \omega) e^{-i \omega y} d \omega+\frac{e^{-i \pi(b-1) / 2}}{\sqrt{2 \pi}} \int_{0}^{\infty} \omega^{b-1} M(a, c ;-i \omega) e^{i \omega y} d \omega
\end{aligned}
$$

If $a, c \in \mathbb{R}$, then $|M(a ; c ; i x)| \leq C|x|^{\max (-a, a-c)}$ [20] (6.13(2)). The conditions on the parameters for absolute convergence of the above integrals are $0<b<\min (a, c-a)$, so the inverse Fourier transform exists. We can rewrite the last integral as:

$I=\frac{e^{i \pi(b-1) / 2}}{\sqrt{2 \pi}} \lim _{\varepsilon \downarrow 0} \int_{0}^{\infty} \omega^{b-1} M(a, c ; i \omega) e^{-\omega(\varepsilon+i y)} d \omega+\frac{e^{-i \pi(b-1) / 2}}{\sqrt{2 \pi}} \lim _{\varepsilon \downarrow 0} \int_{0}^{\infty} \omega^{b-1} M(a, c ;-i \omega) e^{-\omega(\varepsilon-i y)} d \omega$

The integrals are known [20] (6.10(5)). It follows:

$$
I=\frac{\Gamma(b)}{\sqrt{2 \pi}} \lim _{\varepsilon \downarrow 0} \frac{e^{i \pi(b-1) / 2}}{(i y+\varepsilon)^{b}} F\left(\begin{array}{c}
a, b \\
c
\end{array} ; \frac{i}{\varepsilon+i y}\right)+\frac{\Gamma(b)}{\sqrt{2 \pi}} \lim _{\varepsilon \downarrow 0} \frac{e^{-i \pi(b-1) / 2}}{(\varepsilon-i y)^{b}} F\left(\begin{array}{c}
a, b \\
c
\end{array} ; \frac{-i}{\varepsilon-i y}\right)
$$

Taking the limit gives:

$$
I=-i \frac{\Gamma(b)}{\sqrt{2 \pi}} \frac{1}{(y-i 0)^{b}} F\left(\begin{array}{c}
a, b \\
c
\end{array} ; \frac{1}{y-i 0}\right)+i \frac{\Gamma(b)}{\sqrt{2 \pi}} \frac{1}{(y+i 0)^{b}} F\left(\begin{array}{c}
a, b \\
c
\end{array} ; \frac{1}{y+i 0}\right)
$$

Because there is a cut, we distinguish three possibilities. 
I. For $y>1$, there remains:

$$
I=i \frac{\Gamma(b)}{\sqrt{2 \pi}} \frac{1}{y^{b}} F\left(\begin{array}{c}
a, b \\
c
\end{array} ; \frac{1}{y}\right)-i \frac{\Gamma(b)}{\sqrt{2 \pi}} \frac{1}{y^{b}} F\left(\begin{array}{c}
a, b \\
c ; \frac{1}{y}
\end{array}\right)=0
$$

II. For $0<y<1$, there remains:

$$
I=i \frac{\Gamma(b)}{\sqrt{2 \pi}} \frac{1}{(y+i 0)^{b}} F\left(\begin{array}{c}
a, b \\
c
\end{array} ; \frac{1}{y+i 0}\right)-i \frac{\Gamma(b)}{\sqrt{2 \pi}} \frac{1}{(y-i 0)^{b}} F\left(\begin{array}{c}
a, b \\
c
\end{array} ; \frac{1}{y-i 0}\right)
$$

We use [20] (2.10(4)). This formula gives:

$$
\begin{aligned}
F\left(\begin{array}{c}
a, b \\
c
\end{array} ;\right) & =\frac{\Gamma(c) \Gamma(c-a-b)}{\Gamma(c-a) \Gamma(c-b)} \frac{1}{z^{a}} F\left(a, a+1-c a+b+1-c ; 1-\frac{1}{z}\right)+ \\
& +\frac{\Gamma(c) \Gamma(a+b-c)}{\Gamma(a) \Gamma(b)} \frac{(1-z)^{c-a-b}}{z^{c-a}} F\left(\begin{array}{c}
c-a, 1-a \\
c+1-a-b
\end{array} ; 1-\frac{1}{z}\right)
\end{aligned}
$$

Application gives:

$$
\begin{aligned}
I & =i \frac{\Gamma(b)}{\sqrt{2 \pi}} \frac{1}{y^{b}} \frac{\Gamma(c) \Gamma(a+b-c)}{\Gamma(a) \Gamma(b)} y^{c-a}\left(1-\frac{1}{y+i 0}\right)^{c-a-b} F\left(\begin{array}{c}
c-a, 1-a \\
c+1-a-b
\end{array} ; 1-y\right)- \\
& -i \frac{\Gamma(b)}{\sqrt{2 \pi}} \frac{1}{y^{b}} \frac{\Gamma(c) \Gamma(a+b-c)}{\Gamma(a) \Gamma(b)} y^{c-a}\left(1-\frac{1}{y-i 0}\right)^{c-a-b} F\left(\begin{array}{c}
c-a, 1-a \\
c+1-a-b
\end{array} ; 1-y\right)
\end{aligned}
$$

After a little computation, it follows:

$$
I=\sqrt{2 \pi} \frac{\Gamma(c)}{\Gamma(a) \Gamma(1-a-b+c)}(1-y)^{c-a-b} F\left(\begin{array}{c}
c-a, 1-a \\
c+1-a-b
\end{array} ; 1-y\right)
$$

Application of [13] (15.8.1) gives at last:

$$
I=\sqrt{2 \pi} \frac{\Gamma(c)}{\Gamma(a) \Gamma(1-a-b+c)} y^{a-b}(1-y)^{c-a-b} F\left(\begin{array}{c}
1-b, c-b \\
c+1-a-b
\end{array} ; 1-y\right)
$$

III. For $y<0$, there remains:

$$
I=i \frac{\Gamma(b)}{\sqrt{2 \pi}} \frac{1}{(-y)^{b}}\left[e^{-i \pi b}-e^{i \pi b}\right] F\left(\begin{array}{c}
a, b \\
c
\end{array} ; \frac{1}{y}\right)=\frac{\sqrt{2 \pi}}{\Gamma(1-b)} \frac{1}{(-y)^{b}} F\left(\begin{array}{c}
a, b \\
c
\end{array} ; \frac{1}{y}\right)
$$

Application of [13] (15.8.1) gives:

$$
I=\frac{\sqrt{2 \pi}}{\Gamma(1-b)} \frac{1}{(1-y)^{b}} F\left(\begin{array}{c}
b, c-a \\
c
\end{array} ; \frac{1}{1-y}\right)
$$

Gathering the results completes the proof. 


\section{B. Appendix: Proof of Theorem 6.5}

In this section, we prove:

$$
\begin{aligned}
& I(k)=\frac{1}{\Gamma(-\nu)} \int_{1}^{\infty} y^{k} \frac{1}{(1+y)^{\nu+1}} F\left(\begin{array}{c}
\nu+1, c-a \\
c
\end{array} ; \frac{2}{1+y}\right) d y+ \\
& +\frac{\Gamma(c)}{2^{c-\nu-1} \Gamma(a) \Gamma(c-a-\nu)} \int_{-1}^{1}(-y)^{k}(1+y)^{a-\nu-1}(1-y)^{c-a-\nu-1} F\left(\begin{array}{c}
-\nu, c-\nu-1 \\
c-a-\nu
\end{array} ; \frac{1-y}{2}\right) d y=0
\end{aligned}
$$

with conditions for the parameters: $a, c \in \mathbb{R}$ and $k+\nu<a<c-\nu$ and $0<k<\nu<a+1$.

To prove this formula, we apply [13] (15.8.1) on the hypergeometric function in the second integral and get:

$$
\begin{aligned}
I(k) & =\frac{1}{\Gamma(-\nu)} \int_{1}^{\infty} y^{k} \frac{1}{(1+y)^{\nu+1}} F\left(\nu+1, c-a ; c ; \frac{2}{1+y}\right) d y+ \\
& +\frac{\Gamma(c)}{2^{c-a} \Gamma(a) \Gamma(c-a-\nu)} \int_{-1}^{1}(-y)^{k}(1-y)^{c-a-\nu-1} F\left(c-a, 1-a ; c-a-\nu \frac{1-y}{2}\right) d y=0
\end{aligned}
$$

Transformation of the first integral with $z=\frac{2}{1+y}$ and the second integral with $z=\frac{1-y}{2}$ gives:

$$
\begin{aligned}
I(k) & =\frac{1}{2^{\nu} \Gamma(-\nu)} \int_{0}^{1}\left(\frac{2-z}{z}\right)^{k} z^{\nu-1} F(\nu+1, c-a ; c ; z) d z+ \\
& +\frac{\Gamma(c)(-1)^{k}}{2^{\nu} \Gamma(a) \Gamma(c-a-\nu)} \int_{0}^{1}(1-2 z)^{k} z^{c-a-\nu-1} F(c-a, 1-a ; c-a-\nu z) d z=0
\end{aligned}
$$

We write:

$$
I(k)=\frac{1}{2^{\nu} \Gamma(-\nu)} I_{1}+\frac{\Gamma(c)(-1)^{k}}{2^{\nu} \Gamma(a) \Gamma(c-a-\nu)} I_{2}=0
$$

To compute the first integral, we use:

$$
\int x^{a-1} F(a+1, b ; c ; x)=\frac{1}{a} x^{a} F(a, b ; c ; x)
$$

Partial integration gives:

$$
\begin{aligned}
I_{1} & =\int_{0}^{1}\left(\frac{2-z}{z}\right)^{k} z^{\nu-1} F(\nu+1, c-a ; c ; z) d z= \\
& =\frac{1}{\nu}\left[(2-z)^{k} z^{\nu-k} F(\nu, c-a ; c ; z)\right]_{0}^{1}+\frac{2 k}{\nu} \int_{0}^{1}\left(\frac{2-z}{z}\right)^{k-1} z^{\nu-2} F(\nu, c-a ; c ; z) d z
\end{aligned}
$$

For $k<\nu$, the first term converges. There remains:

$$
I_{1}=\frac{1}{\nu} F(\nu, c-a ; c ; 1)+\frac{2 k}{\nu} \int_{0}^{1}\left(\frac{2-z}{z}\right)^{k-1} z^{\nu-2} F(\nu, c-a ; c ; z) d z
$$

After doing $k-1$ times partial integration, there remains:

$$
\begin{aligned}
I_{1} & =\frac{\Gamma(k+1)}{\Gamma(\nu+1)} \sum_{m=0}^{k-1} 2^{m} \frac{\Gamma(\nu-m)}{\Gamma(k+1-m)} F(\nu-m, c-a ; c ; 1)+ \\
& +2^{k} \Gamma(k+1) \frac{\Gamma(\nu-k+1)}{\Gamma(\nu+1)} \int_{0}^{1} z^{\nu-k-1} F(\nu-k+1, c-a ; c ; z)
\end{aligned}
$$


The remaining integral is known [19] (399(5)):

$$
\int_{0}^{1} y^{\rho-1}(1-y)^{\sigma-1} F(\alpha, \beta ; \gamma ; y) d y=\frac{\Gamma(\rho) \Gamma(\sigma)}{\Gamma(\rho+\sigma)}{ }_{3} F_{2}(\alpha, \beta, \rho ; \gamma, \rho+\sigma ; 1)
$$

with conditions: $\rho>0, \sigma>0, \gamma+\sigma-\alpha-\beta>0$. Application gives:

$$
\begin{aligned}
I_{1}= & \frac{\Gamma(k+1)}{\Gamma(\nu+1)} \sum_{m=0}^{k-1} 2^{m} \frac{\Gamma(\nu-m)}{\Gamma(k+1-m)} F(\nu-m, c-a ; c ; 1)+ \\
& +2^{k} \Gamma(k+1) \frac{\Gamma(\nu-k)}{\Gamma(\nu+1)} F(c-a, \nu-k ; c ; 1)= \\
= & \frac{\Gamma(k+1)}{\Gamma(\nu+1)} \sum_{m=0}^{k} 2^{m} \frac{\Gamma(\nu-m)}{\Gamma(k+1-m)} F(\nu-m, c-a ; c ; 1)
\end{aligned}
$$

with conditions: $1+a>\nu-k>0$. For the quotient of the Gamma functions, it follows:

$$
\frac{\Gamma(\nu-m)}{\Gamma(k+1-m)}=-\frac{\Gamma(m-k)}{\Gamma(m+1-\nu)} \frac{\Gamma(\nu) \Gamma(1-\nu)}{\Gamma(k) \Gamma(1-k)}
$$

Substitution in (49) gives:

$$
I_{1}=-\frac{\Gamma(-\nu)}{\Gamma(-k)} \sum_{m=0}^{k} 2^{m} \frac{\Gamma(m-k)}{\Gamma(m+1-\nu)} F(\nu-m, c-a ; c ; 1)
$$

The hypergeometric function is known if $0<m \leq k<a-\nu$. There remains at last:

$$
\begin{aligned}
I_{1} & =-\frac{\Gamma(c)}{\Gamma(a)} \frac{\Gamma(-\nu)}{\Gamma(-k)} \sum_{m=0}^{k} \frac{\Gamma(m-k) \Gamma(m-\nu+a) \Gamma(m+1)}{\Gamma(m+1-\nu) \Gamma(m+c-\nu)} \frac{2^{m}}{m !}= \\
& =-\frac{\Gamma(c)}{\Gamma(a)} \frac{\Gamma(-\nu)}{\Gamma(1-\nu)} \frac{\Gamma(a-\nu)}{\Gamma(c-\nu)}{ }_{3} F_{2}(-k, 1, a-\nu ; 1-\nu, c-\nu ; 2)
\end{aligned}
$$

The integral $I_{2}$ is known [19] (20.2(6)):

$$
\begin{aligned}
\int_{0}^{1} x^{\gamma-1}(1-x)^{\rho 1}(1-z x)^{-\sigma} & F(\alpha, \beta ; \gamma ; x) d x= \\
= & \frac{\Gamma(\gamma) \Gamma(\rho) \Gamma(\gamma+\rho-\alpha-\beta)}{\Gamma(\gamma+\rho-\alpha) \Gamma(\gamma+\rho-\beta)}(1-z)^{-\sigma} \\
& { }_{3} F_{2}\left(\rho, \sigma, \gamma+\rho-\alpha-\beta ; \gamma+\rho-\alpha, \gamma+\rho-\beta ; \frac{z}{z-1}\right)
\end{aligned}
$$

with conditions: $\gamma>0, \rho>0, \gamma+\rho-\alpha-\beta>0$ and $|\arg (1-z)|<\pi$. Application gives:

$$
\begin{aligned}
I_{2} & =\int_{0}^{1}(1-2 z)^{k} z^{c-a-\nu-1} F(c-a, 1-a ; c-a-\nu ; z) d z= \\
& =\frac{\Gamma(c-a-\nu) \Gamma(a-\nu)}{\Gamma(1-\nu) \Gamma(c-\nu)}(-1)^{k}{ }_{3} F_{2}(1,-k, a-\nu ; 1-\nu, c-\nu ; 2)
\end{aligned}
$$


with conditions: $c-a-\nu>0, a-\nu>0$. After substitution of (50) and (51) in (48), there remains at last:

$$
\begin{aligned}
I(k) & =-\frac{1}{2^{\nu}} \frac{\Gamma(c)}{\Gamma(a)} \frac{\Gamma(a-\nu)}{\Gamma(1-\nu) \Gamma(c-\nu)}{ }_{3} F_{2}(-k, 1, a-\nu ; 1-\nu, c-\nu ; 2)+ \\
& +\frac{1}{2^{\nu}} \frac{\Gamma(c)}{\Gamma(a)} \frac{\Gamma(a-\nu)}{\Gamma(1-\nu) \Gamma(c-\nu)}{ }_{3} F_{2}(1,-k, a-\nu ; 1-\nu, c-\nu ; 2)=0
\end{aligned}
$$

This completes the proof.

\section{References}

1. Miller, K.S.; Ross, B. An Introduction to the Fractional Calculus and Fractional Differential Equations; John Wiley \& Sons: Hoboken, NJ, USA. 1993.

2. Samko, S.G.; Kilbas, A.A.; Marichev, O.I. Fractional Integrals and Derivatives; Gordon and Breach: Yverdon, Switzerland, 1993.

3. Kilbas, A.A.; Srivastava, H.M.; Trujillo, J.J. Theory and Applications of Fractional Differential Equations; Elsevier: Amsterdam, Netherlands. 2006.

4. Ortigueira, M.D. Fractional Calculus for Scientists and Engineers; Springer Verlag: Berlin, Germany, 2011.

5. Tenreiro Machado, J.A.; Kiryakova, V.; Mainardi, F. Recent history of fractional calculus. Commun Nonlinear Sci. Numer. Simul. 2011, 16, 1140-1153.

6. Podlubny, I. Geometric and physical interpretation of fractional integration and fractional differentiation. Fract. Calc. Appl. Anal. 2002, 5, 367-386.

7. Tenreiro Machado, J.A. Fractional derivatives: Probability interpretation and frequency response of rational approximations. Commun. Nonlinear Sci. Numer. Simul. 2009, 14, 3492-3497.

8. Diekema, E.; Koornwinder, T.H. Differentiation by integration using orthogonal polynomials, a survey. J. Approx. Theory 2012, 164, 637-667.

9. Chen, D.; Chen, Y. Digital Fractional Order Savitzky-Golay Differentiator. IEEE Trans. Circuits Syst. II Express Briefs 2011, 58, 758-762.

10. Liu, D.-Y.; Gibaru, O.; Perruquetti, W.; Laleg-Kirati, T. Fractional order differentiation by integration with Jacobi polynomials. In Proceedings of the 51st IEEE Conference on Decision and Control, Maui, HI, USA, 10-13 December 2012; pp. 624-629. arXiv.org/abs/:1209.1192.

11. Diekema, E. The fractional orthogonal difference with applications, 2015. Available online: http://www.mdpi.com/journal/mathematics/special_issues/fractional-calculus (accessed on 22 April 2015).

12. Andrews, G.E.; Askey, R.; Roy, R. Special Functions; Cambridge University Press: Cambridge, UK, 1999.

13. Olver, F.W.J.; Lozier, D.W.; Boisvert, R.F.; Clark, C.W. NIST Handbook of Mathematical Functions; Cambridge University Press: Cambridge, UK. 2010; http://dimf.nist.gov/.

14. Koekoek, R.; Lesky, P.A.; Swarttouw, R.F. Hypergeometric Orthogonal Polynomials and Their q-Analogues; Springer-Verlag: Berlin, Germany. 2010.

15. Tseng, C.C.; Pei, S.C.; Hsia, S.C. Computation of fractional derivatives using Fourier transform and digital FIR differentiator. Signal Process. 2000, 80, 151-159. 
16. Kiryakova, V. Generalized Fractional Calculus and Applications; Wiley \& Sons: Hoboken, NJ, USA. 1994.

17. Lavoie, J.L.; Osler, T.J.; Tremblay, R. Fractional Derivatives and Special Functions. SIAM Rev. 1976, 18, 240-268.

18. Zavada, P. Operator of fractional derivative in the complex domain. Commun. Math. Phys. 1998, 192, 261-285.

19. Erdélyi, A. Tables of Integral Transforms; McGraw-Hill: New York, NY, USA.1954; Volume I \& II.

20. Erdélyi, A. Higher Transcendental Functions; McGraw-Hill: New York, NY, USA. 1953; Volume I \& II.

(c) 2015 by the author; licensee MDPI, Basel, Switzerland. This article is an open access article distributed under the terms and conditions of the Creative Commons Attribution license (http://creativecommons.org/licenses/by/4.0/). 